\title{
Large-Scale Bose-Einstein Condensation in an Atomic Gas by Applying an Electric Field
}

\author{
Pei-Lin You \\ Institute of Quantum Electronics, Guangdong Ocean University, Zhanjiang, China
}

\section{Email address:}

youpeli@163.com

\section{To cite this article:}

Pei-Lin You. Large-Scale Bose-Einstein Condensation in an Atomic Gas by Applying an Electric Field. American Journal of Modern Physics. Vol. 7, No. 4, 2018, pp. 121-130. doi: 10.11648/j.ajmp.20180704.11

Received: May 29, 2018; Accepted: June 21, 2018; Published: July 13, 2018

\begin{abstract}
Large-scale Bose-Einstein condensation (BEC) of cesium atoms has been observed ( $\mathrm{T}=343 \mathrm{~K})$. The technical bottleneck of BEC is very small trapping volume $\left(10^{-8} \mathrm{~cm}^{3}\right)$, which made the number of condensed atoms still stagnant (less than $10^{7}$ ), much smaller than normal condensation (more than $10^{13}$ ), large-scale BEC has never been observed. In BEC experiment, scientists have applied magnetic field (used to trap atoms) and laser (used to cool atoms), but never considered applying electric field, because they think that all kinds of atoms are non-polar atoms. The breakthrough of the bottleneck lies in the application of electric field. In theory, despite $6 \mathrm{~s}$ and $6 \mathrm{p}$ states of cesium are not degenerate, but Cs may be polar atom doesn't conflict with quantum mechanics because it is hydrogen-like atom. When an electric field was applied, Cs atoms become dipoles, therefore large-scale BEC can be observed. BEC experiment of cesium has been redone. From the entropy $S=0$, critical voltage $V_{c}=78 \mathrm{~V}$. When $\mathrm{V}<\mathrm{V}_{\mathrm{c}}, \mathrm{S}>0$; when $\mathrm{V}>\mathrm{V}_{\mathrm{c}}, \mathrm{S}<0$, phase transition occurred. When $\mathrm{V}=370 \mathrm{~V}$, condensates contained up to $2.71 \times 10^{17}$ atoms, a forty percent improvement over previous results. This BEC is a second-order phase transition because entropy doesn't show discontinuity, from Wikipedia. It is also equivalent to a quantum phase transition. The permanent dipole moment (PDM) of Cs atom has been accurately measured: $\mathrm{d}_{\mathrm{Cs}}=[1.84 \pm 0.15$ (stat) \pm 0.11 (syst) $] \times 10^{-8} \mathrm{e} . \mathrm{cm}$. The PDM doesn't arise from the nuclear spin but arises from asymmetrical charge distribution, and it doesn't violate both time reversal and parity symmetry. The maximum induced dipole moment is $\mathrm{d}_{\text {ind }} \leq 2.12 \times 10^{-13} \mathrm{e} . \mathrm{cm}$, which can be ignored. BEC has three main features: BEC is macroscopic occupation of the ground state of the system; BEC is condensation in momentum space; Bose gas would undergo a phase transition. Our experiment is an ideal BEC because it conforms to the three features. BEC belongs to the condensation of quantum gas, when $\mathrm{V}>\mathrm{V}_{\mathrm{c}}$, almost all Cs atoms (bosons) are in exactly the same state, according to Feynman, "the quantum physics is the same thing as the classical physics", so our classical theory can explain BEC experiment satisfactorily. Ultra-low temperature is to make Bose gas phase transition, we used critical voltage to achieve phase transition, ultra-low temperature is no longer necessary. Five innovative formulas were first reported in the history of physics, the publication of this article marking mankind will enter a new era of polar atoms.
\end{abstract}

Keywords: Presence of Polar Atom, Entropy of Bose Gas, Order Parameter of BEC, BEC as Second-Order Phase Transition, BEC as Quantum Phase Transition, Three Main Features of BEC, Non-zero PDM of Cs Atom

\section{Introduction}

Bose-Einstein condensation (BEC) is a very active research field in condensed matter physics and material science. A suitable example of BEC is provided by a gas of an appropriate density of alkali-metal atoms (density range from $10^{13}$ to $10^{15} \mathrm{~cm}^{-3}$ ). All stable alkali species--- Li (1), Na (2), K (3), Rb (4), and Cs (5) ---have been condensed. In the past, there are two ways to achieve phase transition: given atomic density, lower the temperature of Bose gas, making $\mathrm{T}<\mathrm{T}_{\mathrm{c}}$ $=\frac{2 \pi \hbar^{2}}{k m}\left(\frac{n}{2.612}\right)^{2 / 3} ;$ or given temperature, increase the density of Bose gas, making $\mathrm{n}>\mathrm{n}_{\mathrm{c}}$ (the critical density $\mathrm{n}_{\mathrm{c}}$ $\left.=2.612\left(\frac{m k T}{2 \pi \hbar^{2}}\right)^{1 / 3}\right)$. But the latter method is not successful, because the density of Bose gas can't be increased indefinitely, and therefore the laser cooling is now widely used. The key requirement is ultra-low temperatures-less than a millionth of a kelvin above absolute zero. Unlike the liquid-gas transition, 
this transition is not driven by interactions between particles [6]. Although many advanced technologies are used, but the vast majority of atoms are randomly oriented, large-scale BEC has never been observed [1-6]. In November 1995, W. Ketterle used a cloud of sodium to create BEC with $5 \times 10^{5}$ atoms and won the Nobel Prize in Physics in 2001. He pointed out that "Optical dipole traps provide tight confinement, but have only a very small trapping volume $\left(10^{-8} \mathrm{~cm}^{3}\right)$." [2] Eight years later, in the BEC experiment of cesium atoms, scientists created BEC with $1.6 \times 10^{4}$ atoms [5]. The trapping volume not only did not increase, but decreased to $0.5 \times 10^{-8} \mathrm{~cm}^{3}$, because the density of cesium reached $1.3 \times 10^{13} \mathrm{~cm}^{-3}$, the number of trapping atoms is only $6.5 \times 10^{4}[5]$. This fact shows that the current technical bottleneck is a very small trapping volume $\left(10^{-8} \mathrm{~cm}^{3}\right)$. So the number of condensed atoms is still less than $10^{7}\left(10^{15} \mathrm{~cm}^{-3} \times 10^{-8} \mathrm{~cm}^{3}\right)$. Ketterle stressed that BEC, "Its most striking feature is a macroscopic occupation of the ground state of the system" [2]. The total number of atoms in a rarefied Bose gas is usually greater than $10^{15}$, but only $10^{4}-10^{6}$ of them, can be trapped and cooled [1-6]. This fact shows that scientists still don't know how to truly realize "the macroscopic occupation of the ground state" so far [1-6]. In addition, from Wikipedia, "quantum phase transition describes an abrupt change in the ground state of a many-body system. Such a quantum phase transition can be a second-order phase transition." However, all previous articles failed to prove that the existing BEC experiments belong to second-order phase transition. These two facts reveal the shortcomings of the existing BEC research. Can we find a new way to get out of the present predicament? This article provides a new idea. Contrary to classical phase transitions, quantum phase transitions can only be accessed by varying a physical parameter-such as voltage. So the best way to achieve BEC is to measure capacitance at different voltages, which is a breakthrough discovery. When $\mathrm{V}=370$ volts, the capacitance decreased from $\mathrm{C}=2.2 \mathrm{C}_{0}$ to $\mathrm{C} \approx \mathrm{C}_{0}\left(\mathrm{C}_{0}\right.$ is the vacuum capacitance), this result implies that almost all the $\mathrm{Cs}$ atoms (more than 98\%) were to line up along the field, condensates contained up to $2.71 \times 10^{17}$ atoms(the total number of $\mathrm{Cs}$ atoms is $2.74 \times 10^{17}$ ). The waveform images on the oscilloscope clearly and truly show that large-scale BEC has been observed, this is the condensation in momentum space. The partition function formula(Eq.(1)), entropy formula (Eq.(2)), order parameter formula(Eq.(5)), condensate fraction formula(Eq.(9)) and atomic PDM formula(Eq.(11)) was first published in the history of physics, they constituted a complete theory of polar atoms. On the other hand, despite the relentless search for a non-zero PDM of an atom for more than 50 years, no conclusive results have been obtained so far [25-27]. Now an accurate and meaningful result has been obtained on the PDM of Cs atom. Experimental alkali material with purity $99.95 \%$ was supplied by Strem Chemicals Co., USA.

\section{Our Innovation}

A common view is that all kinds of atoms have no PDM because of their spherical symmetry, and there is no polar atom in nature except polar molecules. However, our research showed that alkali atoms form an exception. If alkali atom is a nonpolar atom, has this conclusion been verified by the experiment? Answer: no! Such experimental results have never been reported in the history of physics! In fact, this deep-rooted traditional concept is an unconfirmed assumption that misleads scientists all over the world. Although this assumption has never been suspected, it must be verified by accurate experiments. The title of the article is "Theoretical and experimental proof of alkali metal atoms as polar atoms", which has been published in Journal of Material Sciences \& Engineering (Impact Factor is 5.447) [7]. Reviewer comments pointed out that "The main goal of this submission is to prove the presence of polar atom in nature. This was done both theoretically and experimentally. The author has experimentally found that most alkaline atoms $(\mathrm{Na}, \mathrm{K}, \mathrm{Rb}$ and $\mathrm{Cs}$ ) are polar atoms. He found this innovation by measuring their capacitance and temperature-dependence of their density. In fact, using Bose-Einstein condensation some decades ago, have shown that slim part of atom can be condensed $\left(10^{7}\right)$ which is by far less than the normal conditions. The present author has modified Bose-Einstein condensation experiment and applied an electric field, which have a great impact on the production technique and on the performance in their monolithic type reactor system. The submission contributes to an increased knowledge either in theoretical or experimental parts. The subject of the above-mentioned submission is interesting and I suggest publishing the manuscript." The fact shows that our innovative idea and experiments have been accepted by the scientific community.

The ways to achieve the macroscopic occupation of the ground state as follows. Our innovation lies in the application of an electric field, we don't need a magnetic field and a laser. When the electric field was applied, alkali atoms become dipoles, they tend to align with the field because of the torque it experiences. The magnitude of the torque is $\tau=d E \sin \theta$, and the electric potential energy is $\varepsilon=-d E \cos \theta$. When $\theta=0$, the dipole is in equilibrium, and its potential energy is a minimum. But atomic collisions tend to disarrange the dipoles. From the entropy $\mathrm{S}=0$, critical voltage $\mathrm{V}_{\mathrm{c}}=78$ volts. When $\mathrm{V}<\mathrm{V}_{\mathrm{c}}$, many atoms are in random directions, this state has high entropy $\mathrm{S}>0$ and $\mathrm{C}>>\mathrm{C}_{0}$; when $\mathrm{V}>\mathrm{V}_{\mathrm{c}}$, the atoms become aligned with the field, this state has low entropy $\mathrm{S}<0$, phase transition occurred. When $\mathrm{V}>>\mathrm{V}_{\mathrm{c}}, \mathrm{C} \approx \mathrm{C}_{0}$, almost all the $\mathrm{Cs}$ atoms were to line up along the field, which is the macroscopic occupation of the ground state. These atoms have the same momentum, and this is condensation in momentum space.

This article is based on two previous articles, and this fact greatly enhanced the credibility of this work $[8,9]$. In March 2016, when $\mathrm{V}=390$ volts, the electric field produced condensates of $2.51 \times 10^{17}$ sodium atoms, and large-scale BEC has been observed [8]. The title of this article is "Bose-Einstein condensation in a vapor of sodium atoms in an electric field". After a rigorous peer review, this article has been published in a professional journal (Physica B: condensed matter physics) [8]. Reviewer comment pointed out that "The author presented a good idea (using the critical 
voltage) to observe the BEC. Moreover, the author shows that the ultra-low temperature is not a necessary condition to verify the existence of BEC. This paper is interesting and certainly deserves publication in the journal." This reviewer, V. Konotop, has published three theoretical articles about BEC in Physical Review E [10-12]. This fact indicates that the peer review is authoritative, and the conclusion is convincing. In August 2016, condensates contained up to $1.93 \times 10^{17}$ cesium atoms, and this article was accepted and published by Journal of Material Sciences \& Engineering (OMICS Group)[9]. However, the two articles don't relate to entropy curve, order parameters and the classification of phase transitions, thus the essential features of BEC can't be found. Recently, BEC experiments of $\mathrm{Cs}$ atoms have been redone, and the experimental data have been updated. These rich contents and new results will be reported in this article.

\section{Theoretical Breakthrough}

In theory, the $6 s$ and $6 p$ states of cesium atoms are not degenerate, so the expectation value of PDM is zero: $<\psi_{\mathrm{E}}|e r| \psi_{\mathrm{E}}>=0(\mathrm{~d}=$ er is the dipole moment operator) [13]. However, many physicists think that the quantum state $\psi_{\mathrm{E}}$ doesn't describe an individual particle but an ensemble of particles with the same energy [13-15]. In the abstract of an article entitled "The statistical interpretation of quantum mechanics", this article has been published in Reviews of Modern Physics, L. E. Ballentine stressed that " the quantum state description to apply only to an ensemble of similar prepared systems, rather than supposing, as is often done, that it exhaustively represents an individual physical system. Most of the problems associated with the quantum theory of measurement are artifacts of the attempt to maintain the latter interpretation." [14] D. J. Griffiths also stresses that "The expectation value is the average of repeated measurements on an ensemble of identically prepared systems, not the average of repeated measurements on one and the same system." [16] It convincingly proved that $\left\langle\psi_{\mathrm{E}}|\mathrm{er}| \psi_{\mathrm{E}}\right\rangle=0$ only means that the average PDM of large number of cesium atoms is zero, but doesn't mean that the PDM of individual Cs atom is zero.

The hydrogen atom is a typical example. "The shift in the energy levels of an atom in an electric field is known as the Stark effect." [13] Normally the effect is quadratic in the field intensity, which corresponds to an induced electric dipole moment. L.D. Landau once stated that "the hydrogen atom forms an exception; here the Stark effect is linear in the field." "The energy levels of the hydrogen atom, unlike those of other atoms, undergo a splitting proportional to the field (the linear Stark effect)." [17] Evidently, the linear effect corresponds to a PDM. This effect showed that the first excited state of hydrogen atom $(n=2)$ has the PDM of magnitude $3 \mathrm{ea}_{0}=1.59 \times 10^{-8} \mathrm{e} . \mathrm{cm}$ ( $\mathrm{a}_{0}$ is the Bohr radius) [17-19]. Landau once stated that "The presence of the linear effect means that, in the unperturbed state $\psi_{21 \mathrm{~m}}$, the hydrogen atom has a dipole moment whose mean value is $3 \mathrm{ea}_{0} . "$ [17]. L.I. Schiff also stated that "It is also possible, as in the case of the hydrogen atom, that unperturbed degenerate states of opposite parities can give rise to a permanent electric dipole moment." [18] Griffiths emphasizes that "Spin is irrelevant to this problem, so ignore it, and neglect the fine structure." "Notice that the results are independent of the applied field, ---evidently hydrogen in its first excited state can carry a permanent electric dipole moment." [16] The four wave functions of the unperturbed state are $\psi_{200}, \psi_{210}, \psi_{211}$ and $\psi_{21-1}$. That is, $\mathrm{d}\left(\psi_{200}\right)=\mathrm{d}\left(\psi_{210}\right)=\mathrm{d}\left(\psi_{211}\right)=\mathrm{d}\left(\psi_{21-1}\right)=3 \mathrm{ea}_{0}$. However, although $\psi_{21 \mathrm{~m}}$ is four-fold degenerate, quantum mechanical calculations indicated that the expectation value of PDM is zero: $\left\langle\psi_{21 \mathrm{~m}}|\mathrm{er}| \psi_{21 \mathrm{~m}}>=0\right.$ ! That is, $\mathrm{d}\left(\psi_{200}\right)=\mathrm{d}\left(\psi_{210}\right)=$ $\mathrm{d}\left(\psi_{211}\right)=\mathrm{d}\left(\psi_{21-1}\right)=0$. Evidently, the zero result is inconsistent with above conclusion. Up to now, no quantum mechanical textbook explains this contradictory result. This fact shows that individual hydrogen $(\mathrm{n}=2)$ has a non-zero PDM, but quantum mechanics can't obtain this non-zero result in any way. It proved that $\left\langle\psi_{21 \mathrm{~m}}|\mathrm{er}| \psi_{21 \mathrm{~m}}\right\rangle=0$ only means that the average PDM of large number of hydrogen is zero, but an individual hydrogen $(n=2)$ may have a non-zero PDM.

Recall that alkali atoms having only one valence electron in the outermost shell can be described as hydrogen-like atoms [19]. So $<\psi_{\mathrm{E}}|\operatorname{er}| \psi_{\mathrm{E}}>=0$ only means that the average PDM of large number of alkali atoms is zero, but doesn't mean that PDM of individual alkali atom is zero. Alkali atoms (including cesium) may be polar atom doesn't conflict with quantum mechanics. Individual alkali atom is or is not polar atom must be determined by experiments.

The entropy and order parameter clearly described the order-disorder transition, which are the two most important concepts in BEC. The entropy of a system is a measure of the disorder of molecular or atomic motion. This is a thermodynamic quantity that is not easily measured. However, using a combination of theoretical and experimental techniques, entropy can in fact be accurately estimated. Consider a system composed of $\mathrm{N}$ cesium atoms, which are placed in an electric field $E, \theta$ is the angle between $d$ and $E$. Note that the collision between cesium atoms is always through their mass centers, and therefore the atomic nucleus has no contribution to the rotational energy of the atom. When orientation polarization occurs, its rotational energy can be neglected. The potential energy of the atom is $\varepsilon=-d E \cos \theta$. The orientation of cesium atoms can be changed continuously in the field. Note that $\beta=1 / \mathrm{kT}$ and the chemical potential $\mu \approx 0$ [6], the single-particle partition function is given by

$$
Z=\int_{0}^{2 \pi} d \varphi \int_{0}^{\pi} e^{-\beta \varepsilon} \sin \theta d \theta=\int_{0}^{2 \pi} d \varphi \int_{0}^{\pi} e^{d E \cos \theta / k T} \sin \theta d \theta=2 \pi k T\left(e^{d E / k T}-e^{-d E / k T}\right) / d E
$$

All thermodynamic quantities, including entropy and order parameter, can be obtained from it. The entropy of a system is given by $\mathrm{S}=N k\left(\ln Z+T \frac{\partial}{\partial T} \ln Z\right) \quad$ [6]. Let the coefficient $a=$ $\mathrm{dE} / \mathrm{kT}=\mathrm{dV} / \mathrm{kTH}$, we obtain 


$$
\mathrm{S}(a)=N k\left[\ln 2 \pi e\left(e^{a}-e^{-a}\right) / a-a \operatorname{coth} a\right]
$$

The critical coefficient is $a_{c}=2 \pi e \approx 17.08$. Corresponds to $a_{c}$, the voltage $\mathrm{V}_{\mathrm{c}}$ is called the critical voltage. When $a>>1, e$

$-{ }^{a} \approx 0$ and coth $a \approx 1$, we obtain a simplified formula

$$
\mathrm{S}=N k \ln 2 \pi \text { ela }
$$

The entropy formula contains two fundamental constants in nature $(\pi \approx 3.14159$ and $\mathrm{e} \approx 2.71828$ ), and there was ample reason to believe that it reflects the objective laws of BEC (see Figure 1).

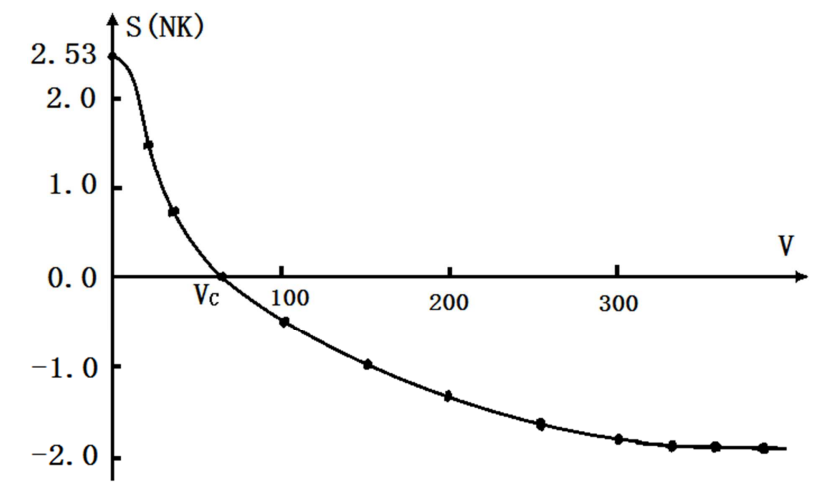

Figure 1. $S-V$ curve shows that BEC is a typical second-order phase transition, because the entropy doesn't show a discontinuity, this fact proved that it is also a quantum phase transition. Its inflection point is found: $V_{p}=$ 8.7 volts.

Ehrenfest proposed a classification of phase transitions [6]. $\mathrm{S}-\mathrm{V}$ curve shows that our BEC is a second-order phase transition, because the entropy doesn't show a discontinuity and its inflection point is found (Figure 1). The fact shows that the $\mathrm{BEC}$ experiment has no latent heat, it is continuous phase transition. Now we find the inflection point of $\mathrm{S}(a)$. From the second order derivative of Eq.(2) is zero, we obtain

$$
\mathrm{S}^{\prime \prime}(a)=\operatorname{coth}^{2} a+2 a \operatorname{coth} a+1 / a^{2}-2 a \operatorname{coth}^{3} a-1=0
$$

Note that coth $a=\left(e^{a}+e^{-a}\right) /\left(e^{a}-e^{-a}\right)$, obviously, there is no analytical solution to this equation. From $\mathrm{S}^{\prime \prime}(1.9111860)=$ $-6 \times 10^{-9}<0, S^{\prime \prime}(1.9111861) \approx 0$ and $S^{\prime \prime}(1.9111862)=2 \times 10^{-8}>$ 0 , we obtain its inflection point is $a_{\mathrm{p}}=1.9111861 \approx 1.91$. The voltage of the inflection point is $\mathrm{V}_{\mathrm{p}}=8.7$ volts (for cesium) (see Figure 1).

Order parameter is a measure of the degree of order across the boundaries in a phase transition system; it normally ranges between zero in one phase and non-zero in the other phase. An example of an order parameter is the net magnetization in a ferromagnetic system undergoing a phase transition. For liquid/gas transitions, the order parameter is the difference of the densities. So the order parameter of BEC is

$$
\mathrm{P}=n k T \frac{\partial}{\partial E} \ln Z=\mathrm{n} d \mathrm{~L}(a)
$$

where $\mathrm{P}$ is the polarization (the average dipole moment per unit volume), $\mathrm{n}$ is the density of alkali gas, $d$ is the PDM of atom, $\mathrm{L}(a)=\operatorname{coth} a-1 / a$ is Langevin function.
On the other hand, if a material is composed of polar molecules, the polarization also can be computed by the method of statistical mechanics. In order to avoid confusion with the differential symbol $d, d_{0}$ represents the PDM of a molecule. In an electric field $\mathrm{E}=\mathrm{E} \mathrm{e}_{\mathrm{z}}$, the potential energy of a polar molecule is $U=-d_{0} E \cos \theta$. In the heat equilibrium state, the number of molecules at $\theta$ per unit solid angle is proportional to $e^{-U / k T}$, the average dipole moment points in the direction of $\mathrm{e}_{\mathrm{z}},<\mathrm{d}_{0}>=$ $\mathrm{d}_{0}<\cos \theta>\mathrm{e}_{\mathrm{z}}[20]$. The average value of $\cos \theta$ is

$$
\begin{aligned}
<\cos \theta>=\mathrm{f} \int_{0}^{\pi} \cos \theta \exp \left(d_{0} E \cos \theta / k T\right) \sin \theta d \theta=\mathrm{L}(\mathrm{a}), \mathrm{f}= \\
{\left[\int_{0}^{\pi} \exp \left(d_{0} E \cos \theta / k T\right) \sin \theta d \theta\right]^{-1} }
\end{aligned}
$$

where $\mathrm{f}$ is normalization constant. If $\mathrm{n}$ is the density of polar gas, the polarization is given by [20]

$$
\mathrm{P}=\mathrm{n} d_{0}<\cos \theta>=\mathrm{n} d_{0} \mathrm{~L}(a)
$$

Eq.(5) miraculously coincides with the result derived from the statistical mechanics of electromagnetics, which shows that our theory is self-consistent [20]. The creation of ordinal parameter formula is a milestone in the history of BEC, because this formula has not been deduced by any previous BEC article or work [1-12]. $<\cos \theta>=\mathrm{L}(a)$ shows that $\mathrm{L}(a)$ equals the percentage of alkali atoms lined up along the electric field, it equals the condensing fraction of BEC.

How to separate polar and non-polar substances (molecules or atoms) experimentally?

Variation of capacitance with temperature offers a means of separating polar and nonpolar atom experimentally, but no one has done this discriminating experiment until now. If alkali atom is non-polar atom, its capacitance should be independent of temperature, because the nucleus is located at the center of the electron cloud. Conversely, if alkali atom is a polar atom, its capacitance is related to temperature. The electric susceptibility is defined as $\chi_{\mathrm{e}}=\mathrm{C} / \mathrm{C}_{0}-1$. For polar atoms or molecules, the electric susceptibility is given by $\chi_{\mathrm{e}}=\mathrm{n} \alpha+\mathrm{nd}$ $\mathrm{L}(a) / \varepsilon_{0} \mathrm{E}$, where $\alpha$ is the atomic or molecular polarizability. When $a<<1, \mathrm{~L}(a) \approx a / 3$, you can get a familiar formula. [21]

For polar atom $\chi_{\mathrm{e}}=\mathrm{n} \alpha+\mathrm{nd}^{2} / 3 \mathrm{k} \varepsilon_{0} \mathrm{~T}$, for nonpolar atom $\chi_{\mathrm{e}}=\mathrm{n} \alpha(8)$

\section{Experiments and Interpretation}

Experiment 1: accurate measurement of the density of cesium gas.

Chemists think that the measurement of the density of gases is a difficult problem. In the textbook of physical chemistry experiment, the experiment of measuring molecular dipole moment is introduced in detail, and this difficulty in measuring the density of gas is particularly stressed. Two coaxial cylinders constitute a traditional cylindrical capacitor. However, because of the irregular orientation of alkali atoms (including cesium) in the electric field, it is impossible to use this capacitor to carry out the BEC experiment (see Figure 3). 
In order to achieve BEC, an ingenious apparatus has been adopted. Our experimental apparatus is a closed glass container resembling a Dewar flask (Figure 2). The internal and external diameters of the container are $\mathrm{D}_{1}=5.70 \mathrm{~cm}$ and $\mathrm{D}_{2}=8.22 \mathrm{~cm}$. The external and internal surfaces are plated with silver, showed by $\mathbf{a}$ and $\mathbf{b}$ respectively (see Figure 2). The length of the silver layer is $\mathrm{L}=28.2 \mathrm{~cm}$. As a more convenient method, replacing plate silver, we can use aluminum foil wrapped the internal and external surface of the glass container, and the two aluminum cylinders constitute a cylindrical capacitor. Note that unlike the traditional cylindrical glass capacitor, the two aluminum cylinders must be posted on the glass. This capacitor is connected in series by two capacitors. One is called $\mathrm{C}^{\prime}$ and contains the Cs gas of thickness $\mathrm{H}_{0}=9.6 \mathrm{~mm}$; the other is called $\mathrm{C}^{\prime \prime}$ and contains the glass medium of thickness $\mathrm{h}=1.5 \mathrm{~mm}$. The total capacitance is $\mathrm{C}=\mathrm{C}^{\prime} \mathrm{C}^{\prime \prime} /\left(\mathrm{C}^{\prime}+\mathrm{C}^{\prime \prime}\right)$, where $\mathrm{C}^{\prime \prime}$ and $\mathrm{C}$ can be directly measured. When the capacitor was empty, it was pumped to vacuum pressure $\mathrm{P} \leq 10^{-9}$ torr for 20 hours. The aim of the operation is to remove impurities such as oxygen adsorbed on the inner walls of the container. The vacuum capacitance is $\mathrm{C}_{0}$ $=(56.0 \pm 0.1) \mathrm{pF}$. Next, a small amount of Cs material (about 10 grams) was put into the container, and it is again pumped to $\mathrm{P}$ $\leq 10^{-9}$ torr. A cylindrical capacitor filled with Cs gas and surplus liquid cesium has been obtained (Figure 2). Measuring instrument is a digital capacitance meter (DM6031A made in Shenzhen, China). The measuring voltage is $\mathrm{V}_{0}=1.2$ volts, the measuring frequency was $800 \mathrm{~Hz}$, and the definition was 0.1 $\mathrm{pF}(\mathrm{C} \leq 200 \mathrm{pF}), 1 \mathrm{pF}(200 \mathrm{pF} \leq \mathrm{C} \leq 2 \mathrm{nF})$ or $10 \mathrm{pF}(2 \mathrm{nF} \leq \mathrm{C}$ $\leq 20 \mathrm{nF}$ ). The capacitor is put into a temperature-control stove, and keep it at $\mathrm{T}_{0}=473 \mathrm{~K}$ for 6 hours. Its capacitance becomes $\mathrm{C}_{\mathrm{t}}=(5290 \pm 10) \mathrm{pF}$. The saturated vapor pressure of Cs atoms is $\mathrm{P}=10^{6} .^{949}{ }^{-3833} .^{7 / \mathrm{T}}$ psi $(473 \mathrm{~K} \leq \mathrm{T} \leq 623 \mathrm{~K})$, where 1 $\mathrm{psi}=6894.8 \mathrm{~Pa}$ [22]. Thus the saturated vapor pressure is $\mathrm{P}=481.3 \mathrm{~Pa}$ at $\mathrm{T}_{0}=473 \mathrm{~K}$. From the ideal gas law, the density of cesium gas equals $\mathrm{n}_{0}=\mathrm{P} / \mathrm{kT}_{0}=7.37 \times 10^{16} \mathrm{~cm}^{-3}$. The statistical error is $\left(\Delta \mathrm{n}_{\mathrm{i}} / \mathrm{n}\right)^{2}=(\Delta \mathrm{P} / \mathrm{P})^{2}+(\Delta \mathrm{T} / \mathrm{T})^{2}$, due to $\Delta \mathrm{T}=0.5 \mathrm{~K}$ and $\Delta \mathrm{T} / \mathrm{T} \leq 0.001, \Delta \mathrm{P}=\mathrm{P}\left(\mathrm{T}_{0}+\Delta \mathrm{T}\right)-\mathrm{P}\left(\mathrm{T}_{0}\right)=9.6 \mathrm{~Pa}$ and $\Delta \mathrm{P} / \mathrm{P} \leq 0.02$, so $\Delta n_{i} / n \leq 0.03$. Systematic error is $\Delta n_{j} / n \leq 0.03$, the density of cesium gas is $\mathrm{n}_{0}=[7.37 \pm 0.22$ (stat) \pm 0.22 (syst) $] \times 10^{16} \mathrm{~cm}^{-3}$.

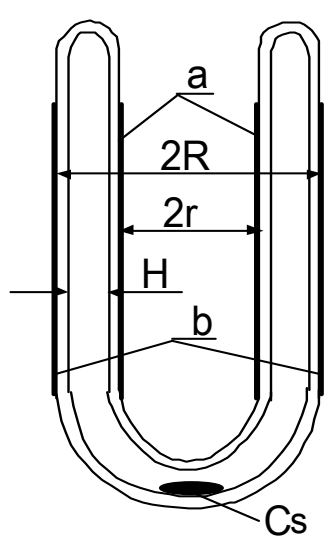

Figure 2. This is an innovative glass cylindrical capacitor resembling a Dewar, which filled with Cs gas and surplus liquid cesium, but in BEC experiment, it keeps a fixed density without liquid cesium. The perfusion method is shown in figure 5.

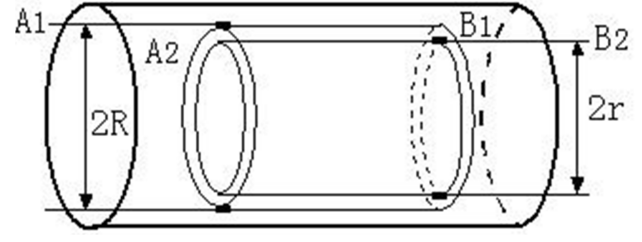

Figure 3. Two coaxial cylinders $A_{1} B_{1}$ and $A_{2} B_{2}$ constitute a traditional cylindrical capacitor. However, it is impossible to carry out BEC experiment with the capacitor because the orientation of alkali atoms (including cesium) is irregular in the electric field.

Experiment 2: accurate measurement of the capacitances of $\mathrm{Cs}$ and $\mathrm{Hg}$ gas at different density.

A cylindrical capacitor fills with Cs gas and surplus liquid cesium, another capacitor fills with $\mathrm{Hg}$ gas and surplus liquid mercury (see Figure 2). Their vacuum capacitance is $\mathrm{C}_{0}=56$ $\mathrm{pF}$ (for $\mathrm{Cs}$ gas) and $\mathrm{C}_{0}^{\prime}=51.8 \mathrm{pF}$ (for $\mathrm{Hg}$ gas) respectively. After fill with $\mathrm{Cs}$ or $\mathrm{Hg}$ materials, the capacitances become $\mathrm{C}_{01}=109.8 \mathrm{pF}$ (for Cs gas) and $\mathrm{C}^{\prime}{ }_{01}=52.1 \mathrm{pF}$ (for $\mathrm{Hg}$ gas). We measure the capacitances and density at different temperatures. The experiment showed that the capacitances of Cs gas increased sharply with the increase of temperature, but the capacitance of $\mathrm{Hg}$ gas, $\mathrm{C}^{\prime}{ }_{01}=52.1 \mathrm{pF}$, remains constant, and $\chi_{\mathrm{e}} \approx 0.006$. Table 1 gives a complete experimental data of cesium. The experimental results are shown in Figure 4.

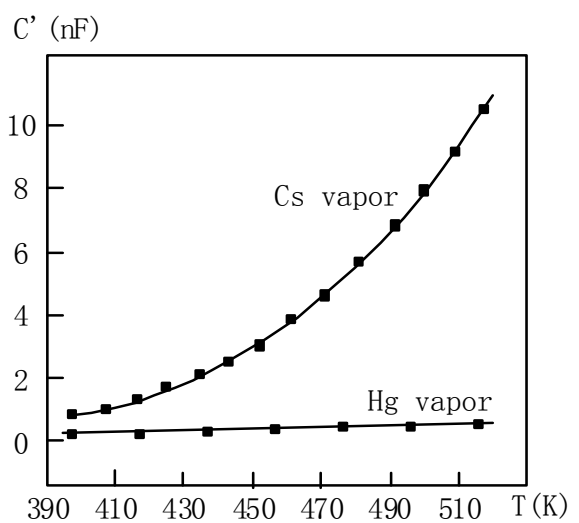

Figure 4. The capacitance of $C s$ vapor varies rapidly with density $(d \neq 0)$, and $\chi_{e}=n d^{2} / 3 k \varepsilon_{0} T$, but the capacitance of $H g$ vapor has not changed $(d=0)$, so cesium is polar atom but mercury is non-polar atom. This is an exciting discovery.

Atomic polarizability of cesium is $\alpha / 4 \pi=59.6 \times 10^{-30} \mathrm{~m}^{3}$, and $\alpha=74.9 \times 10^{-29} \mathrm{~m}^{3}$ [20]. If cesium atom is non-polar atom $(\mathrm{d}=0)$, when $\mathrm{T}=473 \mathrm{~K}$, and $\mathrm{n}_{0}=7.37 \times 10^{22} \mathrm{~m}^{-3}$, the susceptibility of Cs atoms is only $\chi_{\mathrm{e}}=\mathrm{n}_{0} \alpha=5.52 \times 10^{-5}$, the capacitance of Cs vapor is only $\mathrm{C}=56.003 \mathrm{pF}$. However, the experiment shows that when $\mathrm{T}=473 \mathrm{~K}, \chi_{\mathrm{e}}=93.4$, and $\mathrm{C}=5290 \mathrm{pF}$. Obviously, the atomic polarizability and capacitance of $\mathrm{Cs}$ vapor are inconsistent with the experimental results! This experiment proved that cesium atom is a polar atom.

Table 2 gives a complete experimental data of mercury gas. The formula of saturated vapor pressure of mercury atoms is $\mathrm{P}=10^{7}{ }^{752-3065} \cdot{ }^{9 / \mathrm{T}} \mathrm{mmHg} \quad(673 \mathrm{~K} \leq \mathrm{T} \leq 1573 \mathrm{~K}) . \quad$ Because the temperature $(673 \mathrm{~K})$ exceeds the furnace temperature, we use the experimental values of mercury saturated vapor pressure [22]. Atomic polarizability of mercury is $\alpha=(9 / 2) 4 \pi R^{3}=$ 
$19.8 \times 10^{-29} \mathrm{~m}^{3}$, where $\mathrm{R}=1.52 \times 10^{-10} \mathrm{~m}$ is the radius of $\mathrm{Hg}$ atom, $9 / 2$ is the correction of quantum mechanics[20].

When $\mathrm{T}=497 \mathrm{~K}$, and the density of $\mathrm{Hg}$ vapor up to $\mathrm{n}=6.819 \times 10^{23} \mathrm{~m}^{-3}$, but the theoretical value of the susceptibility is only $\chi_{\mathrm{e}}=\mathrm{n} \alpha=1.36 \times 10^{-4}$, the capacitance of $\mathrm{Hg}$ vapor is only $\mathrm{C}=51.81 \mathrm{pF}=\mathrm{C}_{01}^{\prime}$, remains constant (see Table 2).

Table 1. The electric susceptibility of cesium gas at different density $\left(C_{0}\right.$ $=56 p F$.

\begin{tabular}{llllllll}
\hline $\mathbf{( K )}$ & $\mathbf{4 7 3}$ & $\mathbf{4 7 7}$ & $\mathbf{4 8 1}$ & $\mathbf{4 8 5}$ & $\mathbf{4 8 9}$ & $\mathbf{4 9 3}$ & $\mathbf{4 9 7}$ \\
\hline $\mathrm{P}(\mathrm{Pa})$ & 481.3 & 562.8 & 656.4 & 762.9 & 886.1 & 1026 & 1184 \\
$\mathrm{n}\left(10^{23} \mathrm{~m}^{-3}\right)$ & 0.737 & 0.854 & 0.988 & 1.139 & 1.312 & 1.507 & 1.725 \\
$\mathrm{C}(\mathrm{pF})$ & 5290 & 5560 & 5930 & 6380 & 6930 & 7880 & 8960 \\
$\chi_{\mathrm{e}}$ & 93.4 & 98.3 & 104.9 & 112.9 & 122.7 & 139.7 & 159.0 \\
\hline
\end{tabular}

Table 2. The electric susceptibility of mercury gas at different density $\left(C^{\prime}\right.$ $=51.8 p F)$.

\begin{tabular}{llllllll}
\hline $\mathbf{( K )}$ & $\mathbf{4 7 3}$ & $\mathbf{4 7 7}$ & $\mathbf{4 8 1}$ & $\mathbf{4 8 5}$ & $\mathbf{4 8 9}$ & $\mathbf{4 9 3}$ & $\mathbf{4 9 7}$ \\
\hline $\mathrm{P}(\mathrm{Pa})$ & 2304 & 2700 & 3096 & 3492 & 3888 & 4283 & 4679 \\
$\mathrm{n}\left(10^{23} \mathrm{~m}^{-3}\right)$ & 3.528 & 4.10 & 4.662 & 5.215 & 5.758 & 6.292 & 6.819 \\
$\mathrm{C}(\mathrm{pF})$ & 52.1 & 52.1 & 52.1 & 52.1 & 52.1 & 52.1 & 52.1 \\
$\chi_{\mathrm{e}}$ & 0.006 & 0.006 & 0.006 & 0.006 & 0.006 & 0.006 & 0.006 \\
\hline
\end{tabular}

The two tables formed a sharp contrast because Cs atom is polar atom but $\mathrm{Hg}$ atom is non-polar.

Experiment 3: accurate measure capacitance of cesium gas at various voltages.

The best way to realize BEC is to measure the capacitance at different voltages. The experimental instrument is a cylindrical glass capacitor filled with cesium gas at a fixed density $\mathrm{n}_{2}$, and $\mathrm{n}_{2}<\mathrm{n}_{0}$. The experimental temperature is maintained at $343 \mathrm{~K}$. In order to ensure that the capacitor is filled with Cs gas at a fixed density (i.e. ensure the number of Cs atoms remained constant), we adopted an ingenious technique (see Figure 5).

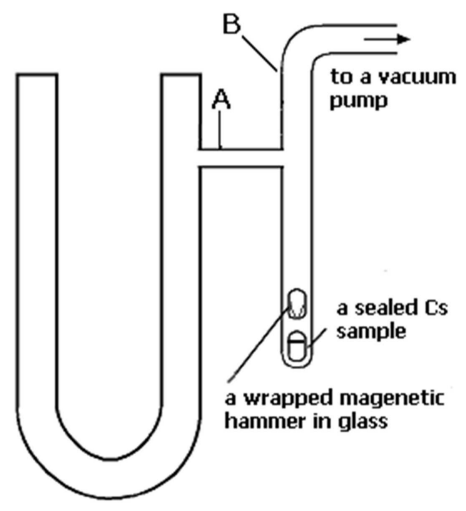

Figure 5. This diagram shows the perfusion method. Let the left capacitor to connect a vacuum pump through a glass tube. In this glass tube, a small magnetic hammer and a sealed cesium sample are put together. When the pump works, the magnetic hammer is raised by a magnet outside the tube. The magnetic hammer is suddenly release, which breaks the bottle sealed cesium sample, and then the tube is sealed at B point. Next step, the two containers are put into a heating furnace, and keep them at a certain temperature for 6 hours. After Cs gas is filled with the capacitor, glass tube is sealed again at $A$ point. Thus a capacitor filled with Cs gas at a fixed density is obtained (i.e. ensure the number of Cs atoms remained constant).

The capacitance is measured by the comparison method, $\mathrm{C}_{2}$ is the capacitor to be measured, its vacuum capacitance is $\mathrm{C}_{20}$ $=(68.0 \pm 0.1) \mathrm{pF}$, and $\mathrm{H}_{2}=7.2 \mathrm{~mm} . \mathrm{C}_{\mathrm{d}}=1878 \mathrm{pF}$ is a standard capacitor, $C_{2}$ and $C_{d}$ are series. Two signals $V_{c}(t)=V_{c o} \cos \omega t$ and $V_{s}(t)=V_{\text {so }} \cos \omega t$ were measured by a two channel digital oscilloscope (Tektronix TDS 210 USA). The signal $V_{c}(t)$ is applied to the capacitor $\mathrm{C}_{2}$, the signal $\mathrm{V}_{\mathrm{s}}(\mathrm{t})$ is applied at both ends of $\mathrm{C}_{2}$ and $\mathrm{C}_{\mathrm{d}}$. Because $\mathrm{C}_{2}$ and $\mathrm{C}_{\mathrm{d}}$ series, so $\left(\mathrm{V}_{\mathrm{s}}-\mathrm{V}_{\mathrm{c}}\right) / \mathrm{V}_{\mathrm{c}}=$ $\mathrm{C}_{2} / \mathrm{C}_{\mathrm{d}}$ and $\mathrm{C}_{2}=\left(\mathrm{V}_{\mathrm{so}} / \mathrm{V}_{\mathrm{co}}-1\right) \mathrm{C}_{\mathrm{d}}$. The voltages $\mathrm{V}_{\mathrm{so}}$ could be adjusted from zero to $800 \mathrm{~V}$. The measurement was started in $0.01 \mathrm{~V}$. When $\mathrm{V}_{1} \leq 0.4 \mathrm{~V}$, the capacitance $\mathrm{C}_{21}=150 \mathrm{pF}$ is approximately constant. When $\mathrm{V}<<\mathrm{V}_{\mathrm{c}}$, the peak difference of the two signals is large, this image means that $\mathrm{C}_{2}>\mathrm{C}_{20}$, this state is far from BEC. For example, when $\mathrm{V}_{\mathrm{so}}=7.08 \mathrm{~V}$, $\mathrm{V}_{\mathrm{co}}=6.6 \mathrm{~V}, \mathrm{C}=(7.08 / 6.6-1) \mathrm{C}_{\mathrm{d}}=136.5 \mathrm{pF}>>\mathrm{C}_{20}$, If almost all the dipoles in a gas were to line up with an external electric field, this effect is called the saturation polarization. The $\mathrm{C}-\mathrm{V}$ curve shows that the saturation polarization of cesium gas is easily observed when $\mathrm{V}>>\mathrm{V}_{\mathrm{c}}$ (Figue 6). When $\mathrm{V}_{\mathrm{so}}=384 \mathrm{~V}, \mathrm{~V}_{2}$ $=\mathrm{V}_{\mathrm{co}}=370 \mathrm{~V}, \mathrm{C}_{22}=(384 / 370-1) \mathrm{C}_{\mathrm{d}}=71.0 \mathrm{pF} \approx \mathrm{C}_{20}$, the oscilloscope suddenly shows that the two signals have the same phase, and they almost overlap. This waveform images clearly and truly show that large-scale BEC has been observed.

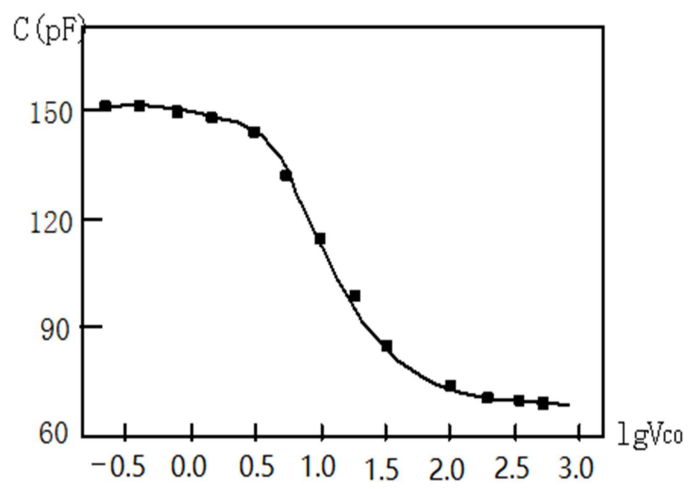

Figure 6. Experimental $C-V$ curve shows that when $V_{1} \leq 0.4$ volts, $C_{21}=$ $150 \mathrm{pF}>>C_{0}$; when $V_{2}=370$ volts, $C_{22}=71.0 \mathrm{pF} \approx C_{20}$. This result implies that almost all the Cs atoms (more than 98.7\%) were to line up along the electric field, condensates contained up to $2.71 \times 10^{17}$ atoms, BEC becomes visible and tangible.

Previous papers usually provide color images of the velocity distribution of trapped atoms, but they are simulated by computer. Many scientists think that these images are "false color images". [23] Unlike them, the waveform images on the oscilloscope show that we created BEC with $2.71 \times 10^{17}$ atoms. This operation needs to repeatedly adjust the voltage and the frequency $(\mathrm{f}=4000--5500 \mathrm{~Hz})$.

The induced susceptibility of Cs is $\chi_{\mathrm{e}}=\mathrm{n}_{2} \alpha \approx 5.3 \times 10^{-7}$ can be neglected, the polarization is defined as $\mathrm{P}=\chi_{\mathrm{e}} \varepsilon_{0} \mathrm{E}=\mathrm{nd}$ $\mathrm{L}(a)$, and therefore $\mathrm{L}(a)$ equals the condensing fraction of BEC. Note that $\mathrm{E}=\mathrm{V} / \mathrm{H}$ and the formula of the parallel-plate capacitor $\varepsilon_{0}=\mathrm{C}_{0} \mathrm{H} / \mathrm{S}$, from $\chi_{\mathrm{e}}=\mathrm{n} \mathrm{d} \mathrm{L}(a) / \varepsilon_{0} \mathrm{E}$ we obtain

Condensate fraction: $\mathrm{L}(a)=a\left(\mathrm{C}-\mathrm{C}_{0}\right) / \eta$ or $\mathrm{C}=\eta \mathrm{L}(a) / a+\mathrm{C}_{0}(9)$

where $\eta=\mathrm{Snd}^{2} / \mathrm{kTH}$ is the capacitance constant. When $\mathrm{V}_{1} \leq$ $0.4 \mathrm{~V}, a_{1}<<1, \mathrm{~L}(a)=a / 3$ and $\eta=3\left(\mathrm{C}_{21}-\mathrm{C}_{20}\right)=246 \mathrm{pF}$. When $\mathrm{V}_{2}$ $=370 \mathrm{~V}, \mathrm{C}_{22}-\mathrm{C}_{20}=3.0 \mathrm{pF}, \mathrm{L}\left(a_{2}\right)=1-1 / a_{2}, a_{2}=\eta \mathrm{L}\left(a_{2}\right) /\left(\mathrm{C}_{22}-\mathrm{C}_{20}\right)$ 
$=81, \mathrm{~L}(81)=0.9876, \mathrm{~V}_{\mathrm{c}}=\mathrm{V}_{2} 2 \pi e / a_{2}=78 \mathrm{~V}$. Notice that Eq. $(9)$ is deduced from the formula of the parallel-plate capacitor $\varepsilon_{0}$ $=\mathrm{C}_{0} \mathrm{H} / \mathrm{S}$, so the cylindrical capacitor must be regarded as an equivalent parallel-plate capacitor with the plate area $\mathrm{S}=\mathrm{C}_{0} \mathrm{H} / \varepsilon_{0}$. In the three experiment, the equivalent area is $\mathrm{S}_{0}=\mathrm{C}_{0} \mathrm{H}_{0} / \varepsilon_{0}=6.072 \times 10^{-2} \mathrm{~m}^{2}$ and $\mathrm{S}_{2}=\mathrm{C}_{20} \mathrm{H}_{2} / \varepsilon_{0}=5.53 \times 10^{-2} \mathrm{~m}^{2}$. Since the density $\mathrm{n}_{2}$ is unknown, from Eq.(9) the density of alkali gas under arbitrary vapor pressure is

$$
\mathrm{n}=\left(\mathrm{C}_{2}{ }_{2}-\mathrm{C}_{20}\right) \mathrm{V}_{2} \mathrm{~L}(a) \mathrm{S}_{0} \mathrm{n}_{0} /\left(\mathrm{C}_{\mathrm{t}}-\mathrm{C}_{0}\right) \mathrm{V}_{0} \mathrm{~L}\left(a_{2}\right) \mathrm{S}_{2}
$$

The density is $\mathrm{n}_{2}=6.893 \times 10^{14} \mathrm{~cm}^{-3}$, where $a=a_{2} \mathrm{~V}_{0} \mathrm{~T}_{2} \mathrm{H}_{2} / \mathrm{T}_{0}$ $\mathrm{H}_{0} \mathrm{~V}_{2}=0.1429$ and $\mathrm{L}(a)=0.0476$. Considering all error, $\mathrm{n}_{2}=$ $[6.89 \pm 0.41$ (stat) \pm 0.34 (syst) $] \times 10^{14} \mathrm{~cm}^{-3}$. The trapping volume reached $\mathrm{S}_{2} \mathrm{H}_{2}=398.2 \mathrm{~cm}^{3}$, the total number of $\mathrm{Cs}$ atoms is $\mathrm{N}=$ $\mathrm{n}_{2} \mathrm{~S}_{2} \mathrm{H}_{2}=2.745 \times 10^{17}$, and the number of condensate atoms is $\mathrm{N}_{\mathrm{c}}=\mathrm{N} \mathrm{L}(a)$.

From the formula (9), atomic PDM can be deduced

$$
\mathrm{d}=\left(\mathrm{C}-\mathrm{C}_{0}\right) \mathrm{V} / \mathrm{L}(\mathrm{a}) \mathrm{n} \mathrm{S}
$$

$\mathrm{d}_{\mathrm{Cs}}=3.47 \mathrm{ea}_{0}=2.95 \times 10^{-29}$ C. $\mathrm{m}=1.84 \times 10^{-8}$ e. cm, where $\left(\mathrm{C}_{\mathrm{t}}-\right.$ $\left.\mathrm{C}_{0}\right)=5234 \mathrm{pF}, \mathrm{V}_{0}=1.2 \mathrm{~V}, \mathrm{~L}(a)=0.0476, \mathrm{~S}_{0}=6.072 \times 10^{-2} \mathrm{~m}^{2}$ and $\mathrm{n}_{0}=7.37 \times 10^{22} \mathrm{~m}^{-3}$. Using BEC data, we obtain the same result: $\mathrm{d}_{\mathrm{Cs}}=2.95 \times 10^{-29}$ C.m $=1.84 \times 10^{-8}$ e.cm, where $\left(\mathrm{C}_{22}-\mathrm{C}_{20}\right)=3 \mathrm{pF}$, $\mathrm{V}_{2}=370 \mathrm{~V}, \mathrm{~L}(a)=0.9876, \mathrm{~S}_{2}=5.53 \times 10^{-2} \mathrm{~m}^{2}$ and $\mathrm{n}_{2}=6.89 \times 10^{20}$ $\mathrm{m}^{-3}$. The statistical error of the measured value is $\Delta \mathrm{d}_{1} / \mathrm{d} \leq 0.08$ due to $\Delta \mathrm{C}_{\mathrm{t}} / \mathrm{C}_{\mathrm{t}} \leq 0.005, \Delta \mathrm{C}_{0} / \mathrm{C}_{0} \leq 0.003, \Delta \mathrm{V}_{0} / \mathrm{V}_{0} \leq 0.03, \Delta \mathrm{n}_{0} / \mathrm{n}_{0} \leq$ $0.06, \Delta \mathrm{S}_{0} / \mathrm{S}_{0} \leq 0.04$. Considering all systematic error, $\Delta \mathrm{d}_{2} / \mathrm{d} \leq$ 0.06 , so $\mathrm{d}_{\mathrm{Cs}}=[1.84 \pm 0.15$ (stat) \pm 0.11 (syst) $] \times 10^{-8} \mathrm{e} . \mathrm{cm}$.

This formula is easy to verify. The magnitude of the PDM is $\mathrm{d}=e \mathrm{r}$. L $(a)$ equals the percentage of Cs atoms lined up along an electric field, $\mathrm{n}$ is its density, $\mathrm{S}$ is the plate area. When the electric field is applied, the change of the charge of the capacitor is $\Delta \mathrm{Q}=\left(\mathrm{C}-\mathrm{C}_{0}\right) \mathrm{V}$. On the other hand, its volume is $\mathrm{SH}$, the total number of oriented atoms of the capacitor is $\mathrm{SH} n \mathrm{~L}(\mathrm{a})$. The number of layers of oriented atoms is $\mathrm{H} / \mathrm{r}$. Because inside the $\mathrm{Cs}$ gas the positive and negative charges cancel out each other, the polarization only gives rise to a net positive charge on one side of the capacitor, and a net negative charge on the opposite side. Therefore $\Delta \mathrm{Q}=\mathrm{SH} \mathrm{nL}(\mathrm{a}) e /(\mathrm{H} / \mathrm{r})=\mathrm{n} \mathrm{S} \mathrm{L}(\mathrm{a}) e \mathrm{r}=\mathrm{n} \mathrm{S} \mathrm{L}(\mathrm{a}) \mathrm{d}=(\mathrm{C}$ $\left.-\mathrm{C}_{0}\right) \mathrm{V}$, so $\mathrm{d}=\left(\mathrm{C}-\mathrm{C}_{0}\right) \mathrm{V} / \mathrm{n} \mathrm{L}(\mathrm{a}) \mathrm{S}$. The maximum induced dipole moment of cesium is $\mathrm{d}_{\text {ind }}=\alpha \varepsilon_{o} \mathrm{E} \leq 2.12 \times 10^{-13} \mathrm{e} . \mathrm{cm}$ can be also neglected. When $\mathrm{V}>>\mathrm{V}_{\mathrm{c}}, \mathrm{C} \approx \mathrm{C}_{0}, \mathrm{~S}<<0$ and $\mathrm{P} \approx \mathrm{P}_{\max }$, Table 3 proved that our experiment is an ideal BEC.

Table 3. Bose-Einstein condensation of cesium atoms at different voltages $(V) T=343 K$.

\begin{tabular}{llllllll}
\hline (volt) & 0.01 & 0.40 & 8.7 & 78 & 220 & 276.4 & 370 \\
$\mathrm{C}(\mathrm{pF})$ & 150 & 150 & 135.1 & 81.6 & 73.0 & 72.0 & 71.0 \\
$a$ & $2.2 \times 10^{-3}$ & 0.087 & 1.91 & 17.08 & 48.2 & 60.5 & 81 \\
$\mathrm{~L}(a)$ & $7.3 \times 10^{-4}$ & 0.029 & 0.5213 & 0.9414 & 0.9776 & 0.9835 & 0.9876 \\
$\mathrm{P}\left(10^{7} \mathrm{e}_{\mathrm{cm}}^{-2}\right)$ & $9.3 \times 10^{-4}$ & 0.037 & 0.6625 & 1.196 & 1.242 & 1.250 & 1.255 \\
$\mathrm{~N}_{\mathrm{c}}\left(\times 10^{17}\right)$ & 0.002 & 0.0801 & 1.431 & 2.584 & 2.683 & 2.70 & 1.271 \\
$\mathrm{~S}(\times \mathrm{Nk})$ & 2.531 & 2.530 & 2.079 & 0 & -1.037 & -1.265 & -1.557 \\
\hline
\end{tabular}

$\mathrm{N}_{\mathrm{c}}$ - The number of condensed atoms, $\mathrm{S}$ - The entropy of Cs gas, $\mathrm{P}$ - The order parameter of BEC. In fact, ultralow temperature is in order to make Bose gas phase transition, this article used the critical voltage to achieve the phase transition, and therefore the ultralow temperature is not necessary for observation of BEC. By mere guesswork, scientists generally believed that alkali atoms don't have PDM, they are nonpolar atom. In fact, this is an idealist philosophy. Our experiment shows that the susceptibility of alkali atoms is $\chi_{\mathrm{e}}=\mathrm{n} \alpha+\mathrm{n} \mathrm{d}^{2} / 3 \mathrm{k} \varepsilon_{0} \mathrm{~T}$, so alkali atom is polar atom. This research proved that the condensate fraction of BEC is $\mathrm{L}(\mathrm{a})=a\left(\mathrm{C}-\mathrm{C}_{0}\right) / \eta$, the PDM of an atom is $\mathrm{d}=\left(\mathrm{C}-\mathrm{C}_{0}\right) \mathrm{V} / \mathrm{L}(\mathrm{a}) \mathrm{S}_{0} \mathrm{n}$, where $\mathrm{C}-\mathrm{C}_{0}$ is the change of the capacitance. If $\left(\mathrm{C}-\mathrm{C}_{0}\right) \neq 0$, the experiment proves the $\mathrm{PDM} d \neq 0$, condensate fraction $\mathrm{L}(\mathrm{a}) \neq 0$, so BEC occurs; conversely, if $\left(\mathrm{C}-\mathrm{C}_{0}\right)=0$, we obtain $\mathrm{d}=0$ and $\mathrm{L}(\mathrm{a})=0$, BEC doesn't occur. Since scientists never measured the capacitance of alkali atoms, so they missed this significant discovery.

Why our classical theory can provide satisfactory explanation for the condensation of quantum gas?

R.P Feynman gave a wonderful demonstration. First, the photon as an example, he once stated that photons are non-interacting bosons and many of them can be in the same state, "analogous to the Schrodinger equation for the electron, the photon equation is just the same as Maxwell's equations for the electromagnetic field, and the wave function is the same as the vector potential A" [24]. Second, in order to explain superconductivity, he remarked that a Cooper pair acts as a boson, many bosons in the same state, " $\psi \psi^{*}$ can be interpreted as the electric charge density", and " $\mathrm{J}$ (the current of probability) becomes directly the electric current density", so the electromagnetic equation can be used instead of Schrodinger equation to explain superconductivity [24]. Similarly, alkali atoms (including cesium) in a rarefied gas are non-interacting bosons, when $\mathrm{V}>>\mathrm{V}_{\mathrm{c}}$, almost all bosons (more than $97 \%$ ) are in exactly the same state. Therefore, according to Feynman, "the quantum physics is the same thing as the classical physics" [24], so our classical theory provided a satisfactory explanation. Attention please, the necessary condition is many alkali atoms are in the same state, an applied electric field can satisfy this requirement. Despite scientists have taken many advanced technologies, however, they can't put the alkali atoms in the same state, so they missed this significant discovery.

\section{Discussion}

\subsection{The Theoretical Evidence That Cesium Atoms Do Not Violate Time Reversal and Parity Symmetry}

Experiments to search for PDM of atoms began half a century ago. In all experiments, they measured the spin resonance frequency $v$ of individual atom by $h v=2 \mu B \pm 2 d E$, where $h$ is Planck's constant, $\mu$ and $d$ is magnetic and electric dipole moments [25-27]. But "experimental searches for PDM have so far yielded null results." [25] This fact shows that this formula is not successful, because it measured the microscopic quantity $d$ by using another microscopic quantity 
$v$. In the case of cesium, famous physicist Weisskopf reports a null result of early experiments: $\mathrm{d}_{\mathrm{Cs}}<3.7 \times 10^{-22} \mathrm{e}$. cm (1968). [27] A representative result as follows: $\mathrm{d}_{\mathrm{Cs}}=(-1.8 \pm 6.7$ (stat) \pm 1.8 (syst)) $\times 10^{-24}$ e.cm [26]. Optical pumping is used to spin polarize the Cs ground state. Evidently, this method is difficult to measure the PDM of Cs atom because its error is 3.7 times as large as the measured value [26]. However, measuring the average kinetic energy of a gas molecule using the temperature is easy: $\mathrm{E}_{\mathrm{k}}=3 \mathrm{kT} / 2$. Similarly, the measurement of the PDM of an atom using the change of the capacitance is easy: $\mathrm{d}=\left(\mathrm{C}-\mathrm{C}_{0}\right) \mathrm{V} / \mathrm{L}(\mathrm{a}) \mathrm{nS}$.

According to quantum theory, an atom in its ground state at most has an extremely small PDM, $d \leq \mathrm{e} \times 10^{-20} \mathrm{~cm}$, it points along the nuclear spin axis and arise mainly from the nuclear spin. Under time reversal the direction of the spin changes, while the direction of the PDM does not change. Therefore, the PDM of an atom would violate time reversal symmetry. By the CPT theorem it also implies a violation of $\mathrm{CP}$ symmetry [25]. In short, if the PDM of atom violates time reversal symmetry, it must have two characteristics: the first is very small, $d<<10^{-20}$ e. cm, and the second is arises from the nuclear spin.

However, the linear Stark effect of hydrogen atom provides a new example. This effect showed that the hydrogen atom $(n=2)$ has a PDM of magnitude $3 \mathrm{ea}_{0}=1.59 \times 10^{-8} \mathrm{e} . \mathrm{cm}$. Since the nuclear spin was completely irrelevant to the calculation of the PDM, and Bohr radius $\left(\mathrm{a}_{0}=0.53 \times 10^{-8} \mathrm{~cm}\right)$ is far greater than the nuclear radius $\left(\mathrm{r}_{0} \approx 10^{-11} \mathrm{~cm}\right)$, so this PDM has nothing to do with the nuclear spin, and only arises from the asymmetrical charge distribution of hydrogen atom. L.D. Landau once stated that the hydrogen atom has a dipole moment whose mean value is $3 \mathrm{ea}_{0}$, "This is in accordance with the fact, in a state determined by parabolic quantum numbers, the distribution of the charges in the atom is not symmetrical about the plane $\mathrm{z}=0$ " [17]. So similar to the first excited state of hydrogen atom, the PDM of Cs atom doesn't arise from the nuclear spin but from asymmetrical charge distribution, and it doesn't violate time reversal and parity symmetry. [25-27]

\subsection{Theoretical Evidence That the Linear Stark Effect of Cesium Atoms Cannot Be Observed}

This is a challenging question. As two concrete examples, first let us deal with the fine structure and the linear Stark shifts of the hydrogen $(n=2)$. The wavenumber of the fine structure of the hydrogen $(n=2)$ is only $0.33 \mathrm{~cm}^{-1}$ for the $\mathrm{H} \alpha$ lines of the Balmer series, where $\lambda=656.3 \mathrm{~nm}$. The splitting is only $\Delta \lambda=0.33 \times\left(656.3 \times 10^{-7}\right)^{2}=0.014 \mathrm{~nm}$, therefore the fine structure is difficult to observe [28]. The linear Stark shift of the hydrogen $(\mathrm{n}=2)$ is proportional to the field intensity: $\Delta \mathrm{W}=\mathrm{d}_{\mathrm{H}} . \mathrm{E}=1.59 \times 10^{-8} \mathrm{E}$ e.cm. When $\mathrm{E}=10^{5} \mathrm{~V} / \mathrm{cm}, \Delta \mathrm{W}=1.59 \times$ $10^{-3} \mathrm{eV}$, this corresponds to a wavenumber of $12.8 \mathrm{~cm}^{-1}$. So the linear Stark shifts is $\Delta \lambda=\Delta \mathrm{W} \lambda^{2} / \mathrm{hc}=12.8 \times\left(656.3 \times 10^{-7}\right)^{2}=0.55$ $\mathrm{nm}$, and the linear Stark shift of the hydrogen $(n=2)$ is easily observed [28]. However, when $\mathrm{V}=370 \mathrm{~V}$, the most field intensity is $\mathrm{E}_{\max }=\mathrm{V} / \mathrm{H}=514 \mathrm{~V} / \mathrm{cm}$. When the external electric field increases to $\mathrm{E}=\mathrm{E}_{\max }$, almost all the Cs atoms (more than $98.7 \%$ ) were to line up along the field, Cs vapor no longer absorb energy, this Stark effect will not occur. if the PDM of cesium is $\mathrm{d}_{\mathrm{Cs}}=1.84 \times 10^{-8} \mathrm{e} . \mathrm{cm}$, and the most splitting of the energy levels is $\Delta \mathrm{W}_{\max }=\mathrm{d}_{\mathrm{Cs}} \mathrm{E}_{\max }=9.46 \times 10^{-6} \mathrm{eV}$. This corresponds to a wavenumber is $\Delta \mathrm{W}_{\max } / \mathrm{hc} \leq 0.076 \mathrm{~cm}^{-1}$. On the other hand, observed values for a line pair of the first primary series of $\mathrm{Cs}$ atom $(\mathrm{Z}=55, \mathrm{n}=6)$ are $\lambda_{1}=894.3 \mathrm{~nm}$ and $\lambda_{2}$ $=852.1 \mathrm{~nm}$ [28]. So the most linear Stark shift of Cs atoms is only $\Delta \lambda=\Delta \mathrm{W}\left(\lambda_{1}+\lambda_{2}\right)^{2} / 4 \mathrm{hc}=0.0057 \mathrm{~nm}$. It is so small, in fact, that a direct observation of the linear Stark shifts of Cs atom is not possible by conventional spectroscopy!

\subsection{The True Meaning of Einstein's Prophecy}

In 1924, S. N. Bose, a physicist from India, wrote to Einstein describing his work on the statistical mechanics of photons. Einstein followed up Bose's work by generalizing it to non-relativistic particles with non-zero mass, and in 1925 he predicted the phenomenon now known as Bose-Einstein condensation. The term 'condensation' often implies a condensation in configuration space. However, for Bose Einstein condensation it is a condensation in the momentum space, because the result of this condensation doesn't produce crystals. In 1925 Einstein pointed out that "I maintain that, in this case, a number of molecules steadily growing with increasing density goes over in the first quantum state (which has zero kinetic energy) while the remaining molecules distribute themselves according to the parameter value $\lambda=1$." "A separation is effected; one part condenses, the rest remains a 'saturated ideal gas' $(A=0, \lambda=1)$." [29] The famous physicist Abraham Pais emphasized that "this is now called Bose-Einstein condensation." [29] If 1/10 is used as "one part" of this gas, now estimate how many atoms are contained in this part of the Bose gas. In order to achieve BEC, the suitable density of Bose gas is $10^{13}-10^{15} \mathrm{~cm}^{-3}$, and the volume of Bose gas is usually greater than $10 \mathrm{~cm}^{3}$, and therefore the total number of atoms in the Bose gas is usually as high as $10^{14}$ or more. So according to Einstein's prediction, the number of condensed atom should be at least greater than $10^{13}$. But in the past, the number of condensed atoms is still very small $\left(<10^{7}\right)$. This fact proves that although physicists have the ability to do BEC experiments, they still need to be more accurate understanding of the true meaning of Einstein's prophecy.

F. Wilczek, he was awarded the Nobel Prize in2004, once stated that "The primary goal of fundamental physics is to discover profound concepts that illuminate our understanding of nature. Discovering new particles, as such, is secondary." [30] Probably, "polar atom" is such a concept.

\section{Conclusion}

\subsection{The Meaning of the Expected Value of Atomic PDM}

In theory, $<\psi_{\mathrm{E}} \mid$ er $\mid \psi_{\mathrm{E}}>=0$ only means that the average PDM of large number of alkali atoms is zero, but doesn't mean that the PDM of individual alkali atom is zero. Despite $6 s$ and $6 p$ states of cesium are not degenerate, but Cs may be polar atom doesn't conflict with quantum mechanics because it is hydrogen-like atom. 


\subsection{All Kinds of Alkali Atoms Are Polar Atom}

All kinds of alkali atoms are non-polar atom, which is an untested hypothesis, and it must be verified by accurate experiments. The capacitance at different temperatures and density are measured, our experiment proved that Cs atom is polar atom, because its electric susceptibility is given by $\chi_{\mathrm{e}}=$ $\mathrm{n} \alpha+\mathrm{n} \mathrm{d} \mathrm{L}(a) / \varepsilon_{0}$ E. But in the past, scientists have never measured the capacitance of alkali atoms, so they missed this significant discovery.

\subsection{Three Main Features of BEC}

BEC has three main features: BEC is a macroscopic occupation of the ground state of the system; BEC is a condensation in momentum space; Bose gas would undergo a phase transition. Due to our experiments are fully in line with these three main features, so although laser cooling techniques are not used, they are an ideal BEC. Ultra-low temperature is in order to make Bose gas phase transition, and we used the critical voltage to describe phase transition, and therefore ultra-low temperature is not necessary for observation of BEC.

\subsection{The Entropy Is the Most Important Concept in BEC}

The entropy of a system is a measure of the disorder of molecular or atomic motion. No doubt, it is the most important concept in BEC. This formula, $\mathrm{S}=N k \ln 2 \pi e / a$, contains two fundamental constants in nature $(\pi \approx 3.14159$ and $\mathrm{e} \approx 2.71828)$, and there was ample reason to believe that it reflects the objective laws of BEC.

\subsection{Polar Atom will Be Candidate for Quantum Computers}

The BEC is a typical second-order phase transition because entropy doesn't show discontinuity, according to Wikipedia, this BEC experiment is also equivalent to a quantum phase transition. Now scientists generally assume that polar molecules may be used as candidate materials for quantum computers. In the future, polar atoms will replace polar molecules as candidate materials for quantum computers because they have a smaller moment of inertia.

\subsection{Theoretical Evidence That Our Theory Is Self-consistent}

Order parameter is a measure of the degree of order across the boundaries in a phase transition system. Order parameter of BEC is the polarization. Entropy and order parameter clearly described order-disorder transition. This formula miraculously coincides with the result derived from the statistical mechanics of electromagnetics, which shows that our theory is self-consistent.

\subsection{Our Classical Theory Provided Satisfactory Explanation}

Alkali atoms (including cesium) in a rarefied gas are non-interacting bosons, when $\mathrm{V} \gg>\mathrm{V}_{\mathrm{c}}$, almost all alkali atoms are in exactly the same state. Therefore, according to Feynman, "the quantum physics is the same thing as the classical physics", so our classical theory provided a satisfactory explanation for BEC experiment.

\subsection{Cs atom Has Non-zero PDM}

The PDM of Cs atom has been accurately measured: $\mathrm{d}_{\mathrm{Cs}}=[1.84 \pm 0.15$ (stat) \pm 0.11 (syst) $] \times 10^{-8}$ e.cm. The PDM doesn't arise from the nuclear spin but arises from asymmetrical charge distribution, and it doesn't violate both time reversal and parity symmetry.

\subsection{We Created Five Innovative Formulas}

Partition function formula, entropy formula, order parameter formula, condensate fraction formula and atomic dipole moment formula not only proves that our experiment is an ideal BEC, but also shows that the complete theory of polar atoms has been obtained. The five innovative formulas were first reported in the history of physics.

\subsection{BEC will Become Enjoyable and Fascinating}

Our experiment is easily repeated in other laboratories, because the details have been described in this article. Once scientists completed these measurements, they will obtain the same results as our experiments. They will discover that a large-scale BEC of cesium atoms is easily observed when an electric field is applied! The publication of the article will lead to a revolution in BEC research and condensed matter physics, marking mankind will enter a new era of polar atoms. We fervently hope that this article will make BEC research to become enjoyable and fascinating. In the face of the energy crisis and the potential threat of climate change, we want to use this article to express our enthusiasm for the readers.

\section{Acknowledgements}

This research was supported by the NSF of Guangdong Province, China (Grant No. 021377). The author thanks to Dr. Yu-Sheng Zhang, Director Xun Chen, Engineer Yi-Quan Zhan (Peking University), Engineer Jia You and our colleagues Rui-Hua Zhou, Xue-Ming Yi and Xin Huang for their help with this work.

\section{References}

[1] F. Schreck, L. Khaykovich, K L. Corwin, et al, Quasipure Bose-Einstein Condensate immersed in a Fermi sea. Phys. Rev. Lett. 2001, 87(8): 080403

[2] K. Davis, M-O. Mewes, M. R. Andrews, N. J. van Druten, D. S Drufee, D. M. Kurn, and W. Ketterle, Bose-Einstein Condensation in a Gas of Sodium Atoms. Phys. Rev. Lett., 1995, 75(22): 3969-3973

[3] G. Modugno, G. Ferrari, G. Roati, RJ. Brecha, A. Simoni, et al., Bose-Einstein Condensation of Potassium Atoms by Sympathetic Cooling, Science 294, 1320 (2001) 
[4] M. D. Barrett, J. A. Sauer, M. S. Chapman, All-optical formation of an atomic Bose-Einstein Condensation. Phys. Rev. Lett. 2001, 87(1): 010404

[5] T. Weber, J. Herbig, M. Mark, H. N gerl, R. Grimm, Bose-Einstein Condensation of Cesium. Science 299, 232(2003)

[6] S. J. Blundell and K. M. Blundell, Concepts in Thermal Physics, (Oxford University Press, Second Edition, 2009) P369, P222

[7] Pei-Lin You, Theoretical and experimental proof of the alkali metal atom as polar atom, J Material Sci Eng 6: 396 (2017). Doi: 10. 4172/2169-0022. 1000396

[8] Pei-Lin You, Bose-Einstein condensation in a vapor of sodium atoms in an electric field, Physica B: condensed matter physics 491(2016) 84-92, http://dx.doi.org/10.1016/j.physb.2016.03.017

[9] Pei-Lin You, Large-Scale Bose-Einstein condensation in a vapor of cesium atoms at normal temperature $(\mathrm{T}=353 \mathrm{~K}), \mathrm{J}$ Material Sci Eng 5: 276 (2016). Doi: 10. 4172/2169-0022. 1000276

[10] Z. Yan, V. Konotop, Exact solutions to three-dimensional generalized nonlinear Schrödinger equations with varying potential and nonlinearities. Phys. Rev. E 80, 036607 (2009)

[11] Z. Yan, V. Konotop, N. Akhmediev, Three-dimensional rogue waves in nonstatio nary parabolic potentials. Phys. Rev. E 82, 036610(2010)

[12] Z. Yan, V. Konotop, A. Yulin, W. Liu, Two-dimensiona superfluid flows in inhomogeneous Bose-Einstein condensates. Phys. Rev. E 85, 016601 (2012)

[13] L. E. Ballentine, Quantum Mechanics a modern development (World Scientific Publishing Co. Pte. Ltd. 1998) P286, P280

[14] L. E. Ballentine, The Statistical Interpretation of Quantum Mechanics. Rev. Mod. Phys., 42358 (1970)

[15] L. E. Ballentine, Yumin Yang, and J. P. Zibin, Inadequacy of Ehrenfest's theorem to characterize the classical regime. Phys. Rev. A. 502854 (1994)
[16] D. J. Griffiths, Introduction to Quantum Mechanics, (Pearson Education, Inc., Second Edition, 2005) P15, P289

[17] L. D. Landau, and E. M. Lifshitz, Quantum Mechanics (Beijing World Publishing Corporation 1999) P285, P289, P290

[18] L. I. Schiff, Quantum Mechanics (New York: McGraw-Hill Book Company 1968) P253, P235, P243

[19] W. Greiner, Quantum Mechanics an introduction (Springer-verlag Berlin/Heidelberg 1994) P213

[20] G. L. Pollack, D. R. Stump, Electromagnetism (Addison Wesley 2002) P189

[21] J. D. Jackson, Classical Electrodynamics (John Wiley \& Sons, Inc. 1999 Third Edition) P165

[22] J. A. Dean, Lange's Handbook of Chemistry (New York: McGraw-Hill, Inc 1998) Table 4.6, 5.3, 5.8

[23] T. Hey and P. Walters, The New Quantum Universe, (Cambridge University Press, $7^{\text {th }}$ printing 2011) P149

[24] R. P. Feynman, R. B. Leighton, \& M. Sands, The Feynman lectures on Physics (Addison-Wesley Publishing Co. 1964) Vol. 3 P21-6

[25] N. Fortson, P. Sandars, and S. Barr, Physics Today, June 2003, 33

[26] S. A. Murthy, D. Krause. Jr., Z. L. Li, and L. R. Hunter, Phys. Rev. Lett. 63, 965 (1989)

[27] M. C. Weisskopf, J. P. Carrico, H. Gould, E. Lipworth, and T. S. Stein, Phys. Rev. Lett. 21, 1645 (1968)

[28] H. Haken, and H. C. Wolf, The Physics of Atoms and Quanta. (Springer-Verlag Berlin Heidelberg 2000) P172

[29] A. Pais, Subtle is the Lord: The Science and the Life of Albert Einstein, (Oxford University Press, Reprinted 2008) P430

[30] F. Wilczek, In search of symmetry lost. Nature, Vol. 433, No. 7023, 20 January (2005) 